\title{
Use of chinese and western over-the-counter medications in Hong Kong
}

\author{
Vincent Chi Ho Chung ${ }^{1 *}$, Chun Hong Lau', Frank Wan Kin Chan', Joyce Hoi Sze You², Eliza Lai Yi Wong ${ }^{1}$, \\ Eng Kiong Yeoh', Sian Meryl Griffiths ${ }^{1}$
}

\begin{abstract}
Benefits of engaging community pharmacists in providing wider primary care are internationally acknowledged; in Hong Kong, however, strategies for harnessing their potential contributions are yet to be launched. Here, community pharmacist and Chinese medicine retailers are responsible for providing western and Chinese over-the-counter (OTC) medications. Patterns of OTC uses reflect the characteristics of populations who rely on community pharmacists and Chinese medicine retailers as their main point of contact with the healthcare system. Analyzing the data from a Hong Kong survey $(n=33,263)$ on self medication and medical consultation patterns, we propose, in this article, an extended role for community pharmacists and Chinese medicine retailers, which entails aspects as follows: (1) referring patients to other medical services where appropriate; (2) providing health education and preventative services; (3) safeguarding the use of Chinese herbal medicines.
\end{abstract}

\section{Background}

In Hong Kong, community pharmacists work independently from medical doctors who often prescribe and dispense medications in a clinical setting. On the other hand, patients often seek first line treatment from community pharmacists [1]. Community pharmacists have long been an underutilized part of the human resources in primary care $[2,3]$ as a result of the interplay between demand, supply and organization factors [4]. In Hong Kong, the use of over-the-counter (OTC) medications is popular in the local population. Previous studies found that $65 \%$ of the respondents used OTC medications [5] and that $32.9 \%$ of outpatients had taken OTC two weeks prior to their visits [6]. The majority of community pharmacists in Hong Kong admitted that they were most frequently asked about OTC [7].

Chinese OTC medications are used as often as their western medicine counterparts in Hong Kong [8]. Unlike pharmacists, tertiary education is not a prerequisite for retailing Chinese medicine OTC [9]. Historically, Chinese medicine retailers worked alongside with Chinese medicine practitioners [10]. Since 1997, Chinese medicine practitioners as a medical profession have

\footnotetext{
* Correspondence: vchung@cuhk.edu.hk

'School of Public Health and Primary Care, The Chinese University of Hong Kong, Shatin, Hong Kong, China

Full list of author information is available at the end of the article
}

been recognized [11] and have become less dependent on Chinese medicine retailers.

This article describes the behavioral patterns of both Chinese and western medical consultations and OTC use in a representative sample of the Hong Kong population. This information will provide timely input for planning pharmacists' and Chinese medicine retailers' future roles within the Hong Kong primary care system [12].

\section{Data from Thematic Household Survey}

Thematic Household Survey (THS) was conducted between November 2005 and March 2006 by the Census and Statistic Department (CSD), Hong Kong [13]. The THS covered the entire land-based population of Hong Kong and interviewed a total of 33,263 non-institutional individuals (response rate: 79.2\%). The interviews were conducted in Cantonese. The sample represents a population of 6,750,652 persons of the general population.

\section{Survey questionnaire on the use of OTC medications}

The questionnaire of THS included a part to solicit information from respondents aged 14 or above on their consultations with western medicine practitioners or Chinese medicine practitioners, as well as their use of

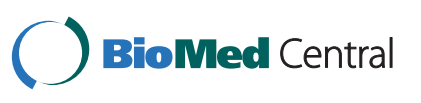

(c) 2010 Chi Ho Chung et al; licensee BioMed Central Ltd. This is an Open Access article distributed under the terms of the Creative Commons Attribution License (http://creativecommons.org/licenses/by/2.0), which permits unrestricted use, distribution, and reproduction in any medium, provided the original work is properly cited. 


\section{Questions on consultations:}

1. In the past 12 months, have you had consultation(s) with a western medicine practitioner in Hong Kong? Please include follow-up consultation.

2. In the past 12 months, have you had consultation(s) with a Chinese medicine practitioner in Hong Kong? Please include follow-up consultation.

\section{Questions on OTC medications use:}

1. In the past 12 months, have you used western medicine OTC, including medicines for external use, due to illness or injury?

2. In the past 12 months, have you ever used Chinese medicine OTC or herbs, including medicines for external use, due to illness or injury?

western or Chinese OTC medications the past 12 months (Figure 1).

Questions about demographic, socioeconomic and health related information were also covered in the THS. These included gender, age, martial status, personal monthly income, education level, self reported chronic disease status as informed by a western medicine practitioner and self perceived level of health and possession of western or Chinese medicine insurance coverage.

\section{Our data analysis}

Analysis of THS data were conducted without imputation of missing data. We focused our multivariate data analysis on respondents who either consulted a western/ Chinese medicine practitioner or used OTC medications in the past year $(n=13,346)$. Sample characteristics were described by cross tabulations of the three patterns (OTC use only, sought consultations only or both) with other demographic, socioeconomic and health related variables. Chi square and one way ANOVA tests were conducted. Multinomial logistic regression analyses were conducted with various demographic, socioeconomic and health related factors as independent variables and patterns of OTC and medical service use as dependent variables. Dependent variables were classified as 'OTC use only' and 'using both OTC and medical services' while 'medical consultation only' was used as a reference. The regression analyses provided adjusted odd ratios for each independent variable, representing its association with the choice of 'OTC only' or 'using both OTC and medical services'. All statistical analyses were performed with SPSS 14.0 (SPSS Inc., Chicago, IL, USA), separately for western and Chinese medicine.

\section{Use of western OTC medications and consultations with western medicine practitioners}

Among all respondents $(n=33,263), 9.4 \%$ used western OTC medications only whereas $41.4 \%$ used both western medicine consultation services and western OTC medications in the previous year. $32.7 \%$ used western medicine consultation only, and $16.6 \%$ used neither western OTC medication nor western medical services (Figure 2). Univariate analysis indicated significant differences among the first three groups in terms of gender, age, education level, health status, chronic disease status, smoking habit, Chinese and/or western medicine insurance and income (Table 1). Multinomial 


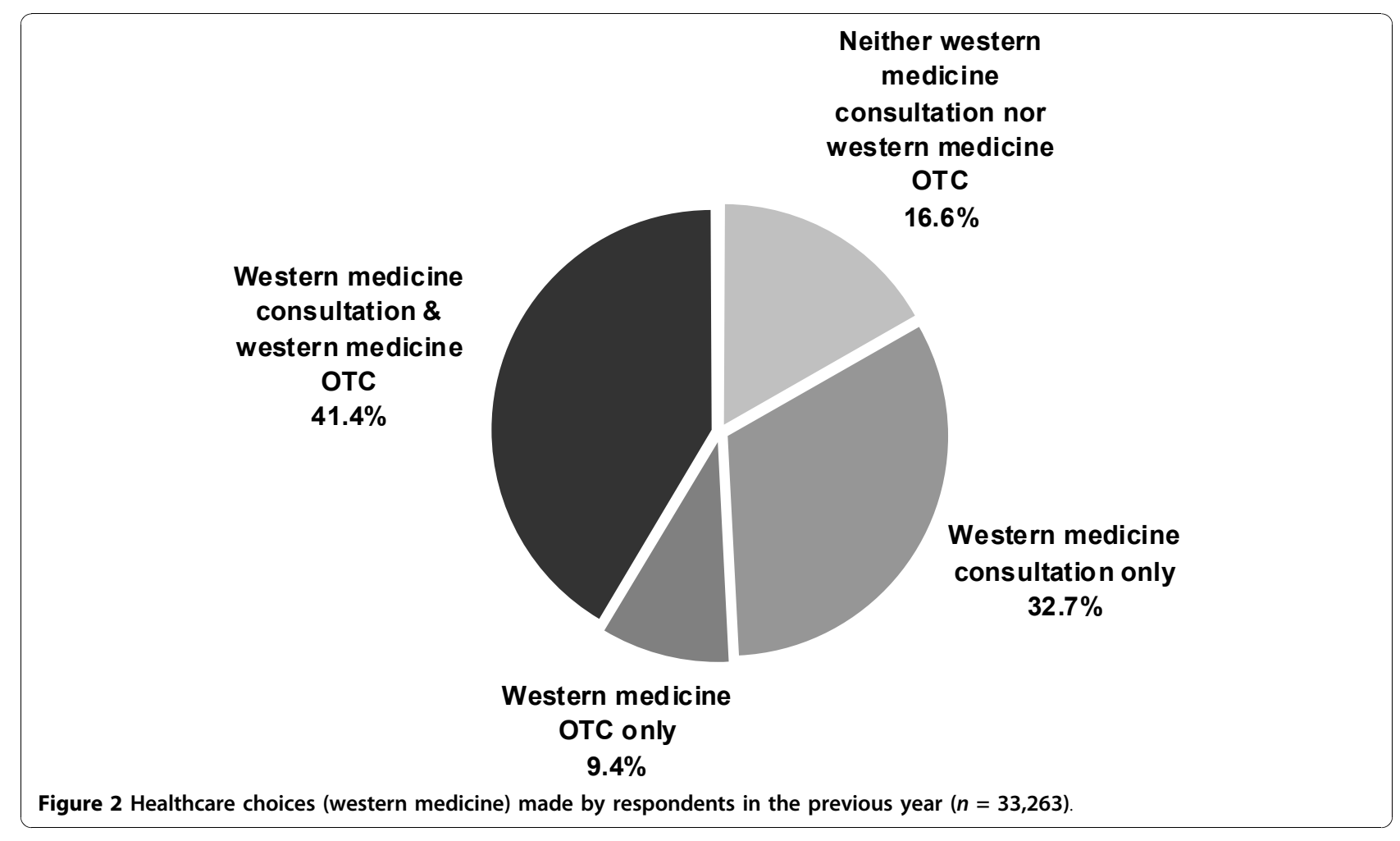

Table 1 Demographic, socioeconomic and health related characteristics among western medical services and OTC medication users

\begin{tabular}{|c|c|c|c|c|c|}
\hline & & $\begin{array}{l}\text { Visited western } \\
\text { medicine } \\
\text { practitioners only (\%) }\end{array}$ & $\begin{array}{l}\text { Used western OTC } \\
\text { medications only } \\
(\%)\end{array}$ & $\begin{array}{l}\text { Consulted western medicine } \\
\text { practitioners and used western OTC } \\
\text { medications (\%) }\end{array}$ & $\begin{array}{l}P \\
\text { values }^{\mathrm{a}}\end{array}$ \\
\hline \multirow[t]{2}{*}{ Gender } & Male & 38.2 & 12.5 & 49.3 & $<0.001$ \\
\hline & Female & 39.6 & 9.9 & 50.5 & \\
\hline \multirow[t]{6}{*}{ Age } & $15-29$ & 36.6 & 12.0 & 51.4 & \\
\hline & $30-39$ & 34.3 & 11.8 & 53.9 & $<0.001$ \\
\hline & $40-49$ & 33.8 & 13.0 & 53.2 & \\
\hline & $50-59$ & 37.5 & 12.3 & 50.2 & \\
\hline & $60-69$ & 45.4 & 8.8 & 45.8 & \\
\hline & $70+$ & 58.6 & 4.4 & 37.0 & \\
\hline \multirow[t]{4}{*}{ Education level } & $\begin{array}{l}\text { Below } \\
\text { primary }\end{array}$ & 54.1 & 5.7 & 40.2 & \\
\hline & Primary & 39.7 & 11.5 & 48.7 & $<0.001$ \\
\hline & Secondary & 34.9 & 12.6 & 52.5 & \\
\hline & Tertiary & 43.6 & 8.4 & 48.1 & \\
\hline \multirow[t]{2}{*}{ Currently married } & Yes & 38.3 & 11.2 & 50.5 & \\
\hline & No & 40.0 & 11.0 & 49.0 & \\
\hline \multirow[t]{3}{*}{ Self reported health } & $\begin{array}{l}\text { Excellent } \\
\text { or very } \\
\text { good }\end{array}$ & 40.7 & 11.4 & 47.9 & $<0.001$ \\
\hline & $\begin{array}{l}\text { Good or } \\
\text { fair }\end{array}$ & 37.5 & 11.5 & 51.1 & \\
\hline & poor & 51.7 & 7.0 & 41.3 & \\
\hline $\begin{array}{l}\text { Self reported chronic disease } \\
\text { status }\end{array}$ & Yes & 51.7 & 1.7 & 46.6 & \\
\hline
\end{tabular}


Table 1 Demographic, socioeconomic and health related characteristics among western medical services and OTC medication users (Continued)

\begin{tabular}{|c|c|c|c|c|c|}
\hline & No & 35.3 & 13.8 & 50.9 & $<0.001$ \\
\hline \multirow[t]{2}{*}{ Drinking habit } & Yes & 37.0 & 13.7 & 49.3 & \\
\hline & No & 39.2 & 10.8 & 50.1 & \\
\hline \multirow[t]{2}{*}{ Current smoker } & Yes & 33.9 & 16.2 & 49.9 & \\
\hline & No & 40.0 & 10.0 & 50.0 & $<0.001$ \\
\hline \multirow{2}{*}{$\begin{array}{l}\text { Moderate exercise }>=2.5 \text { hours/ } \\
\text { week }\end{array}$} & Yes & 43.9 & 9.5 & 46.6 & \\
\hline & No & 37.1 & 11.7 & 51.2 & \\
\hline \multirow{2}{*}{$\begin{array}{l}\text { Possession of western medicine } \\
\text { insurance }\end{array}$} & Yes & 38.2 & 7.5 & 54.3 & \\
\hline & No & 39.2 & 12.4 & 48.5 & $<0.001$ \\
\hline \multirow{2}{*}{$\begin{array}{l}\text { Possession of Chinese medicine } \\
\text { insurance }\end{array}$} & Yes & 36.4 & 7.1 & 56.5 & \\
\hline & No & 39.1 & 11.4 & 49.5 & $<0.001$ \\
\hline Total & & 38.9 & 11.1 & 50.0 & \\
\hline $\begin{array}{l}\text { Mean personal monthly income in } \\
\text { Hong Kong dollars }{ }^{\mathrm{b}} \text { (Standard } \\
\text { Error) }\end{array}$ & & $\$ 10042(\$ 148.64)$ & $\begin{array}{l}\$ 8832 \\
(\$ 221.47)\end{array}$ & $\begin{array}{l}\$ 10117 \\
(\$ 121.21)\end{array}$ & $<0.001$ \\
\hline
\end{tabular}

logistic regression analysis (Table 2) showed that those who only used western OTC medications were more likely to be young adults to middle aged but not over 70, male, primary or secondary educated, having a lower personal monthly income and no insurance coverage for western medical services. They also self reported better perceived health status and being chronic disease free, being a smoker, and not exercising regularly. Those who used both western medicine consultation and western OTC medications demonstrated similar patterns with one exception that their incomes were higher.

Table 2 Association of demographic, socioeconomic and health related characteristics with choices for western medicine consultation and OTC medication

\begin{tabular}{|c|c|c|c|c|}
\hline & $\begin{array}{l}\text { Used western OTC } \\
\text { medications only } \\
\text { Adjusted Odds Ratio } \\
(95 \% \mathrm{Cl})\end{array}$ & $P$ values & $\begin{array}{l}\text { Choice between western medicine } \\
\text { consultations and western OTC } \\
\text { medications Adjusted } \\
\text { Odds Ratios }(95 \% \mathrm{Cl})\end{array}$ & $P$ values \\
\hline \multicolumn{5}{|l|}{ Gender } \\
\hline Female & Reference & & Reference & \\
\hline Male & $1.31(1.17,1.46)$ & $<0.001$ & $1.03(0.96,1.10)$ & NS \\
\hline \multicolumn{5}{|l|}{ Age } \\
\hline $15-29$ & Reference & & Reference & \\
\hline $30-39$ & $1.21(1.02,1.44)$ & 0.032 & $1.09(0.98,1.22)$ & NS \\
\hline $40-49$ & $1.28(1.07,1.53)$ & 0.007 & $1.05(0.94,1.18)$ & NS \\
\hline $50-59$ & $1.14(0.93,1.39)$ & NS & $0.90(0.79,1.02)$ & NS \\
\hline $60-69$ & $0.93(0.73,1.19)$ & NS & $0.73(0.63,0.85)$ & $<0.001$ \\
\hline 70 or above & $0.50(0.38,0.67)$ & $<0.001$ & $0.49(0.42,0.57)$ & $<0.001$ \\
\hline \multicolumn{5}{|l|}{ Education level } \\
\hline Below Primary & Reference & & Reference & \\
\hline Primary & $1.42(1.09,1.84)$ & 0.008 & $1.15(1.01,1.32)$ & 0.047 \\
\hline Secondary & $1.35(1.04,1.75)$ & 0.023 & $1.11(0.97,1.27)$ & NS \\
\hline Tertiary & $0.98(0.72,1.33)$ & NS & $0.84(0.71,0.98)$ & 0.027 \\
\hline \multicolumn{5}{|l|}{ Marital status } \\
\hline Currently married & Reference & & Reference & \\
\hline Not currently married & $1.00(0.88,1.13)$ & NS & $0.95(0.88,1.03)$ & NS \\
\hline Self reported health & & & & \\
\hline
\end{tabular}


Table 2 Association of demographic, socioeconomic and health related characteristics with choices for western medicine consultation and OTC medication (Continued)

\begin{tabular}{|c|c|c|c|c|}
\hline Poor & Reference & & Reference & \\
\hline Good/fair & $1.44(1.15,1.80)$ & 0.001 & $1.44(1.28,1.62)$ & $<0.001$ \\
\hline Excellent/very good & $1.21(0.93,1.57)$ & NS & $1.19(1.03,1.38)$ & 0.021 \\
\hline \multicolumn{5}{|l|}{ Self reported chronic disease status } \\
\hline No & Reference & & Reference: & \\
\hline Yes & $0.10(0.08,0.13)$ & $<0.001$ & $0.82(0.76,0.89)$ & $<0.001$ \\
\hline \multicolumn{5}{|l|}{ Drinking habit } \\
\hline No & Reference & & Reference & \\
\hline Yes & $1.03(0.89,1.21)$ & NS & $0.97(0.88,1.07)$ & NS \\
\hline \multicolumn{5}{|l|}{ Current smoker } \\
\hline No & Reference & & Reference & \\
\hline Yes & $1.56(1.38,1.78)$ & $<0.001$ & $1.12(1.02,1.22)$ & 0.010 \\
\hline \multicolumn{5}{|l|}{ Moderate exercise $>=2.5$ hours/week } \\
\hline No & Reference & & Reference & \\
\hline Yes & $0.81(0.72,0.91)$ & $<0.001$ & $0.86(0.80,0.92)$ & $<0.001$ \\
\hline \multicolumn{5}{|l|}{ Possession of western medicine insurance (\%) } \\
\hline No & Reference & & Reference & \\
\hline Yes & $0.55(0.47,0.63)$ & $<0.001$ & $0.99(0.91,1.07)$ & NS \\
\hline \multicolumn{5}{|l|}{ Possession of Chinese medicine insurance (\%) } \\
\hline No & Reference & & Reference & \\
\hline Yes & $0.91(0.71,1.15)$ & NS & $1.12(0.98,1.27)$ & NS \\
\hline $\begin{array}{l}\text { Monthly personal income } \\
\text { (for every increment of HK\$ 1000, or US\$128.2) }\end{array}$ & $0.98(0.98,0.99)$ & $<0.001$ & $1.00(0.99,1.00)$ & 0.022 \\
\hline
\end{tabular}

Use of Chinese OTC medications and consultations with Chinese medicine practitioners A total of $19.0 \%$ of the population used Chinese OTC medications only whereas $7.2 \%$ used both Chinese medicine consultation and Chinese OTC medication in the previous year. $7.5 \%$ used only Chinese medicine consultation; whereas $66.3 \%$ used neither Chinese OTC medication nor Chinese medicine consultation (Figure 3). Univariate analysis indicated significant differences among the first three groups in terms of gender, age, education level, self reported health status, chronic disease status, smoking, drinking, exercise habit, possession of Chinese and/or western medicine insurance coverage, and income (Table 3). Multinomial logistic regression analysis (Table 4) showed those who only used Chinese OTC medication were more likely to be aged 60 or above, male, to have received no formal education, to have a lower personal monthly income and no insurance coverage for TCM services. Also, they were more likely to report favourable perceived health status, to currently smoke, and to not exercise regularly. Those who used both Chinese medicine consultation and Chinese OTC medication were more likely to be middle aged, to have no insurance coverage for WMD services, and to suffer from chronic diseases.

\section{Discussion and recommendations}

A total of $50.8 \%$ of the Hong Kong population used western OTC medication in the previous year. Assuming that the western OTC medication was obtained from western medicine community pharmacists, we contend that the role of community pharmacists in primary care must not be underestimated. This is vividly illustrated by the fact that $9.4 \%$ of the respondents had no consultation with western medicine practitioners in the previous year but depended on western OTC exclusively for their healthcare. This implies that for these individuals, western medicine community pharmacists might be the only point of contact when they had minor aliments. Therefore, there is a need in promoting and extending the roles of the western medicine community pharmacists in Hong Kong.

A systematic review shows that pharmacists are often perceived by laypersons as drug experts with limited lknowledge on health issues, but customers are generally satisfied with their extended role in providing health advices [14]. In Hong Kong where the culture is unique, local populations' expectation on the extended roles of community pharmacists may be raised to improve patient-oriented community health services. Western medicine community pharmacists' self-perception as a primary care provider is 


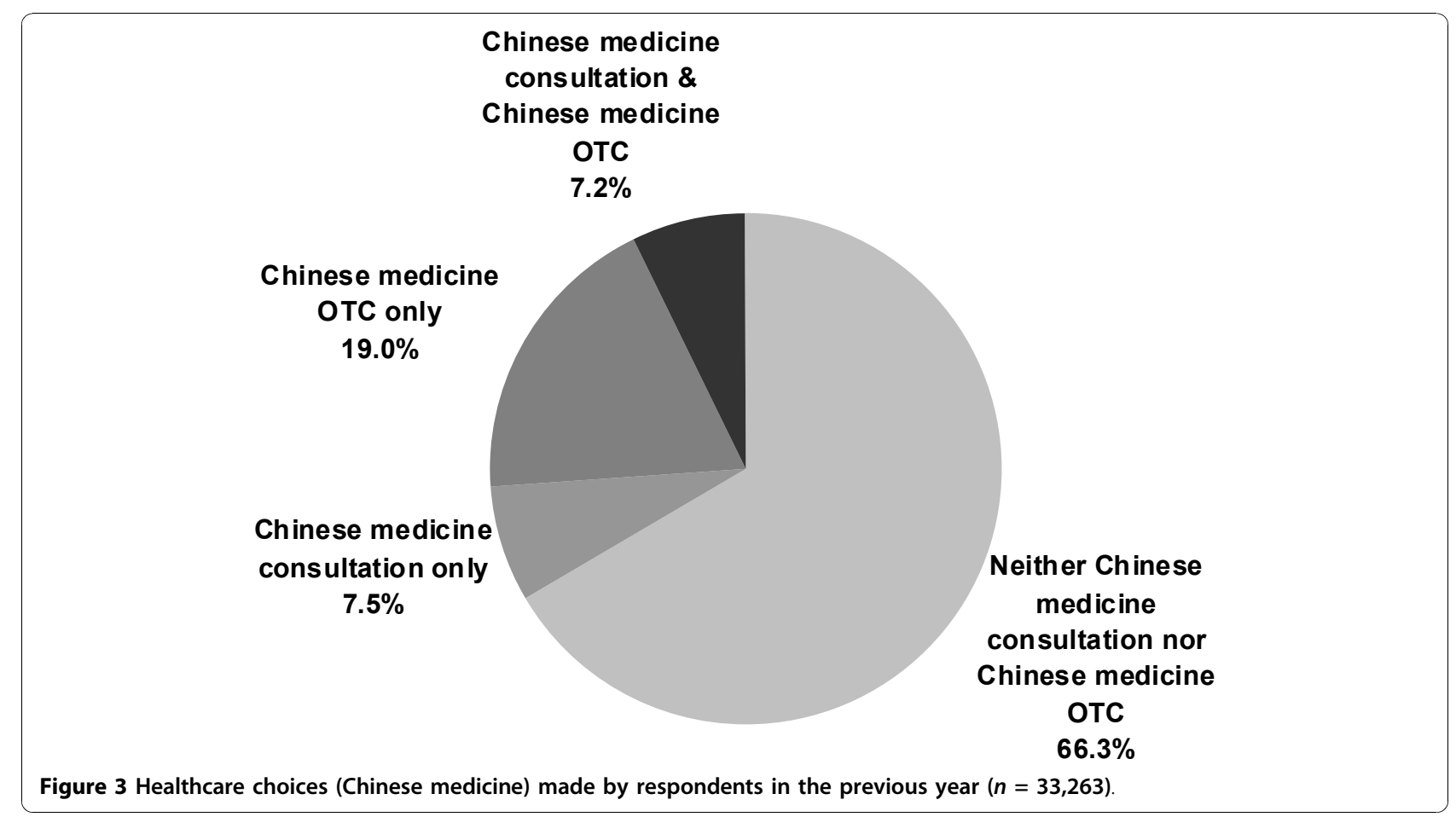

Table 3 Demographic, socioeconomic and health related characteristics among Chinese medical services and OTC medication users

\begin{tabular}{|c|c|c|c|c|c|}
\hline & & $\begin{array}{r}\text { Visited Chinese } \\
\text { medicine } \\
\text { practitioners only (\%) }\end{array}$ & $\begin{array}{r}\text { Used Chinese OTC } \\
\text { medications only } \\
(\%)\end{array}$ & $\begin{array}{r}\text { Consulted Chinese medicine } \\
\text { practitioners and used Chinese OTC } \\
\text { medications (\%) }\end{array}$ & $\begin{array}{l}\mathrm{p}- \\
\text { value }^{\mathrm{a}}\end{array}$ \\
\hline \multirow[t]{2}{*}{ Gender } & Male & 19.9 & 61.9 & 18.2 & $\begin{array}{r}p< \\
0.001\end{array}$ \\
\hline & Female & 23.9 & 51.7 & 24.4 & \\
\hline \multirow[t]{6}{*}{ Age } & $15-29$ & 26.9 & 57.3 & 15.8 & $\begin{array}{rl}p< & < \\
0.001 & 0\end{array}$ \\
\hline & $30-39$ & 29.4 & 50.8 & 19.7 & \\
\hline & $40-49$ & 24.1 & 52.4 & 23.5 & \\
\hline & $50-59$ & 21.9 & 56.0 & 22.2 & \\
\hline & $60-69$ & 16.5 & 57.6 & 25.9 & \\
\hline & $70+$ & 12.7 & 66.1 & 21.3 & \\
\hline \multirow[t]{4}{*}{ Education level } & $\begin{array}{l}\text { Below } \\
\text { primary }\end{array}$ & 12.8 & 66.2 & 21.1 & $\begin{aligned} & p< 0 \\
& 0.001\end{aligned}$ \\
\hline & Primary & 18.5 & 56.8 & 24.7 & \\
\hline & Secondary & 22.8 & 56.5 & 20.6 & \\
\hline & Tertiary & 34.1 & 44.6 & 21.2 & \\
\hline \multirow[t]{2}{*}{ Currently married } & Yes & 22.1 & 55.5 & 22.4 & \\
\hline & No & 22.2 & 57.5 & 20.3 & \\
\hline \multirow[t]{3}{*}{ Self reported health } & $\begin{array}{l}\text { Excellent } \\
\text { or very } \\
\text { good }\end{array}$ & 23.8 & 59.2 & 17.1 & $\begin{aligned} & p<< \\
& 0.001\end{aligned}$ \\
\hline & $\begin{array}{l}\text { Good or } \\
\text { fair }\end{array}$ & 22.0 & 55.8 & 22.2 & \\
\hline & poor & 22.0 & 55.4 & 22.6 & \\
\hline \multirow[t]{2}{*}{$\begin{array}{l}\text { Self reported chronic disease } \\
\text { status }\end{array}$} & Yes & 17.3 & 57.8 & 24.9 & $\begin{array}{c}p< \\
0.001\end{array}$ \\
\hline & No & 23.8 & 55.5 & 20.6 & \\
\hline
\end{tabular}


Table 3 Demographic, socioeconomic and health related characteristics among Chinese medical services and OTC medication users (Continued)

\begin{tabular}{|c|c|c|c|c|c|}
\hline \multirow[t]{2}{*}{ Drinking habit } & Yes & 22.6 & 55.6 & 21.8 & $\begin{aligned} & p<< \\
& 0.001\end{aligned}$ \\
\hline & No & 22.1 & 56.2 & 21.7 & \\
\hline \multirow[t]{2}{*}{ Current smoker } & Yes & 18.5 & 62.1 & 19.4 & $p<$ \\
\hline & No & 22.9 & 54.9 & 22.2 & \\
\hline \multirow[t]{2}{*}{$\begin{array}{l}\text { Moderate exercise }>=2.5 \text { hours } / \\
\text { week }\end{array}$} & Yes & 23.2 & 54.3 & 22.5 & $\begin{aligned} & p<< \\
& 0.001\end{aligned}$ \\
\hline & No & 21.7 & 56.9 & 21.4 & \\
\hline \multirow[t]{2}{*}{$\begin{array}{l}\text { Possession of western medicine } \\
\text { insurance }\end{array}$} & Yes & 30.5 & 48.8 & 20.8 & $\begin{aligned} & p<< \\
& 0.001\end{aligned}$ \\
\hline & No & 19.7 & 58.3 & 22.0 & \\
\hline \multirow[t]{2}{*}{$\begin{array}{l}\text { Possession of Chinese medicine } \\
\text { insurance }\end{array}$} & Yes & 38.6 & 36.1 & 25.4 & $\begin{aligned} & p<< \\
& 0.001\end{aligned}$ \\
\hline & No & 20.8 & 57.7 & 21.4 & \\
\hline Total & & 22.2 & 56.1 & 21.7 & \\
\hline $\begin{array}{l}\text { Mean personal monthly income in } \\
\text { Hong Kong dollars }{ }^{b} \text { (Standard } \\
\text { Error) }\end{array}$ & $\begin{array}{l}\$ 12060 \\
(\$ 350.59)\end{array}$ & $\begin{array}{r}\$ 8180 \\
(\$ 151.10)\end{array}$ & $\begin{array}{r}\$ 9305 \\
(\$ 306.72)\end{array}$ & & $\begin{aligned} & p<< \\
& 0.001\end{aligned}$ \\
\hline
\end{tabular}

Table 4 Association of demographic, socioeconomic and health related characteristics with choices for Chinese medicine consultation and OTC medications

\begin{tabular}{|c|c|c|c|c|}
\hline & $\begin{array}{l}\text { Used Chinese OTC } \\
\text { medications only } \\
\text { Adjusted Odds Ratio } \\
(95 \% \mathrm{Cl})\end{array}$ & $P$ values & $\begin{array}{l}\text { Choice between Chinese medicine } \\
\text { practitioner consultations and } \\
\text { Chinese OTCmedications } \\
\text { Adjusted Odds Ratios }(95 \% \mathrm{Cl})\end{array}$ & $P$ values \\
\hline \multicolumn{5}{|l|}{ Gender } \\
\hline Female & Reference & & Reference & \\
\hline Male & $1.70(1.49,1.93)$ & $<0.001$ & $0.94(0.81,1.10)$ & NS \\
\hline \multicolumn{5}{|l|}{ Age } \\
\hline $15-29$ & Reference & & Reference & \\
\hline $30-39$ & $0.98(0.79,1.23)$ & NS & $1.18(0.89,1.56)$ & NS \\
\hline $40-49$ & $1.10(0.87,1.37)$ & NS & $1.57(1.19,2.08)$ & 0.002 \\
\hline $50-59$ & $1.18(0.92,1.49)$ & NS & $1.49(1.11,2.01)$ & 0.009 \\
\hline $60-69$ & $1.40(1.06,1.86)$ & 0.020 & $2.08(1.48,2.92)$ & $<0.001$ \\
\hline 70 or above & $1.75(1.30,2.35)$ & $<0.001$ & $1.98(1.38,2.84)$ & $<0.001$ \\
\hline \multicolumn{5}{|l|}{ Education level } \\
\hline Below Primary & Reference & & Reference & \\
\hline Primary & $0.66(0.52,0.85)$ & 0.001 & $0.94(0.71,1.26)$ & NS \\
\hline Secondary & $0.69(0.53,0.89)$ & 0.004 & $0.85(0.64,1.15)$ & NS \\
\hline Tertiary & $0.49(0.36,0.66)$ & $<0.001$ & $0.72(0.51,1.03)$ & NS \\
\hline \multicolumn{5}{|l|}{ Marital status } \\
\hline Currently married & Reference & & Reference & \\
\hline Not currently married & $1.09(0.95,1.26)$ & NS & $1.06(0.90,1.26)$ & NS \\
\hline \multicolumn{5}{|l|}{ Self reported health } \\
\hline Poor & Reference & & Reference & \\
\hline Good/fair & $1.38(1.06,1.81)$ & 0.020 & $0.96(0.70,1.33)$ & NS \\
\hline Excellent/very good & $1.28(1.04,1.58)$ & 0.022 & $1.22(0.96,1.55)$ & NS \\
\hline \multicolumn{5}{|c|}{ Self reported chronic disease status } \\
\hline No & Reference & & Reference: & \\
\hline Yes & $1.05(0.90,1.23)$ & NS & $1.28(1.08,1.53)$ & 0.007 \\
\hline
\end{tabular}


Table 4 Association of demographic, socioeconomic and health related characteristics with choices for Chinese medicine consultation and OTC medications (Continued)

\begin{tabular}{|c|c|c|c|c|}
\hline \multicolumn{5}{|l|}{ Drinking habit } \\
\hline No & Reference & & Reference & \\
\hline Yes & $0.86(0.72,1.04)$ & NS & $1.06(0.85,1.31)$ & NS \\
\hline \multicolumn{5}{|l|}{ Current smoker } \\
\hline No & Reference & & Reference & \\
\hline Yes & $1.20(1.01,1.42)$ & 0.034 & $1.12(0.92,1.38)$ & NS \\
\hline \multicolumn{5}{|l|}{ Moderate exercise $>=2.5$ hours/week } \\
\hline No & Reference & & Reference & \\
\hline Yes & $0.82(0.73,0.93)$ & 0.002 & $0.93(0.80,1.08)$ & NS \\
\hline \multicolumn{5}{|l|}{ Possession of western medicine insurance (\%) } \\
\hline No & Reference & & Reference & \\
\hline Yes & $0.87(0.76,1.01)$ & NS & $0.83(0.69,0.99)$ & 0.038 \\
\hline \multicolumn{5}{|l|}{ Possession of Chinese medicine insurance (\%) } \\
\hline No & Reference & & Reference & \\
\hline Yes & $0.52(0.42,0.65)$ & $<0.001$ & $0.93(0.73,1.19)$ & NS \\
\hline $\begin{array}{l}\text { Monthly personal income } \\
\text { (for every increment of HK\$ } 1000 \text {, or US\$ 128.2) }\end{array}$ & $0.98(0.98,0.99)$ & $<0.001$ & $1.00(0.99,1.00)$ & NS \\
\hline
\end{tabular}

NS: Statistically non-significant $(p>0.05)$

also an important factor that determinant the success of their role extension. In Hong Kong, western medicine community pharmacists provide advice about medicines [15-18] rather than addressing the clients' wider determinants of health (only $44 \%$ of community pharmacists consider education activities as one of their main duties) [7]. Appropriate training may help community pharmacists make their primary care practice more evidence-based [19]. Furthermore, stronger incentives and support like such as remuneration should be considered [20].

Other factors such as proximity to other professionals, opportunity for inter-professional communication and access to patients' medical information are essential to integrate pharmacists in the primary care system [20]. The role of medical professionals is a dominant factor in defining, controlling and scoping the work of the allied health professionals [21,22] as extending pharmacists' role in primary care may affect the autonomy and control of the medical professionals [23], particularly the private western medicine practitioners who also dispense medications in their clinics [1]. A stronger linkage between community pharmacists and the primary care team should be established as $39 \%$ of pharmacists did not have frequent communication with other healthcare professionals [7]. A possible option for Hong Kong in the future would be the establishment of integrated prescribing and dispensing service by western medicine practitioners and pharmacists under the same roof in both private and public sectors but this would require much research and harmonization by the government. While this will pose a significant challenge for western medicine, the situation becomes even more complex when the integration with Chinese medicine is taken into account. Previous research has already indicated the needs for western pharmacists to study Chinese medicine [24].

A total of $26.2 \%$ of the respondents reported consuming Chinese OTC medication in the previous year. The majority of them (19\%) reported having no consultation with a Chinese medicine practitioner within the same period. Chinese medicine retailers may have been the only source of guidance on for these respondents. Patients with chronic diseases on western medications are also likely to consume Chinese herbal medicines [25]. Chinese medicine retailers are therefore instrumental in preventing undesirable drug interactions during prescription process. In addition, their role and competence should goes beyond prescription and medication review. All those involved in providing pharmacy services, regardless of Chinese or western medical affiliations, should have a role in gate-keeping other medical services and in promoting health.

\section{Conclusion}

We propose, in this article, an extended role for community pharmacists and Chinese medicine retailers, which entails aspects as follows: (1) referring patients to other medical services where appropriate; (2) providing health education and preventative services; (3) safeguarding the use of Chinese herbal medicines.

\section{Acknowledgements}

This study is funded by the Studies in Health Funding, Health and Food Bureau, Hong Kong SAR Government, China (SHS-P-02). 


\section{Author details}

${ }^{1}$ School of Public Health and Primary Care, The Chinese University of Hong Kong, Shatin, Hong Kong, China. ${ }^{2}$ School of Pharmacy, The Chinese University of Hong Kong, Shatin, Hong Kong, China.

\section{Authors' contributions}

VCHC, CHL and SMG conceived the research idea. CHL conducted the statistical analysis. VCHC interpreted the result and wrote the first draft of the manuscript. FWKC, JHSY, ELYW added critical comments on the interpretations of data and on the manuscript. SMG and EKY supervised the whole research process. All authors read and approved the final manuscript.

\section{Competing interests}

The authors declare that they have no competing interests.

Received: 15 May 2010 Accepted: 10 December 2010 Published: 10 December 2010

\section{References}

1. Mason P: Pharmacy in Hong Kong. Pharm J 2001, 267(7179):911-36.

2. Smith F: The extended role of the community pharmacist: implications for the primary health care team. J Soc Admin Pharm 1990, 7:101-10.

3. Chen J, Britten N: 'Strong medicine': an analysis of pharmacist consultations in primary care. Fam Pract 2000, 17(6):480-3.

4. Hassell K, Rogers A, Noyce P: Community pharmacy as a primary health and self-care resource: a framework for understanding pharmacy utilization. Health Soc Care Community 2000, 8(1):40-9.

5. Lam CLK, Catarivas MG, Munro C, Lauder IJ: Self-medication among Hong Kong Chinese. Soc Sci Med 1994, 39(12):1641-7.

6. Granek-Catarivas M, Lam CLK, Munro C: The use of self-medication among patients attending general practitioners. Hong Kong Pract 1994, 16:488-503.

7. Chan TYK, Lee KKC, Critchley JAJH: The needs and sources of drug information among pharmacists in Hong Kong. J Clin Pharm Ther 1996, 21(5):325-330.

8. Chung V, Wong E, Woo J, Lo SV, Griffiths S: Use of traditional Chinese medicine in Hong Kong Special Administrative Region, China. J Altern Complement Med 2007, 13(3):361-7.

9. Licensing of Chinese Medicines Traders, Chinese Medicine Council of Hong Kong. [http://www.cmchk.org.hk/pcm/eng/idx_licen.htm].

10. Xie YG: History of Chinese Medicine in Hong Kong. Hong Kong: Joint Publishing; 1998

11. Griffiths S, Chung V: Development and regulation of traditional Chinese medicine practitioners in Hong Kong. Perspect Public Health 2009, 129:64.

12. Health and Medical Development Advisory Committee: Building a healthy tomorrow - Discussion paper on the future service delivery model for our health care system. Hong Kong: Hong Kong SAR Government; 2005.

13. Thematic Household Survey Report - Report No. 30, Census and Statistics Department, The Hong Kong SAR Government. [http://www. censtatd.gov.hk/products_and_services/products/publications/ statistical_report/social_data/index_cd_B1130230_dt_latest.jsp].

14. Anderson C, Blenkinsopp A, Armstrong M: Feedback from community pharmacy users on the contribution of community pharmacy to improving the public's health: a systematic review of the peer reviewed and non-peer reviewed literature 1990-2002. Health Expect 2004, 7(3):191-202.

15. Lee WW, Leung PY: Glycemic control and medication compliance in diabetic patients in a pharmacist-managed clinic in Hong Kong. Am J Health Syst Pharm 2003, 60(24):2593-6.

16. Lee SSC, Cheung PYP, Chow MSS: Benefits of individualized counseling by the pharmacist on the treatment outcomes of hyperlipidemia in Hong Kong. J Clin Pharmacol 2004, 44(6):632-9.

17. Cheng CWR, Woo KS, Chan JCN, Tomlinson B, You JHS: Assessing adherence to statin therapy using patient report, pill count, and an electronic monitoring device. Am J Health Syst Pharm 2005, 62(4):411-5.

18. Chan FWH, Wong RSM, Lau WH, Chan TYK, Cheng G, You JHS: Management of Chinese patients on warfarin therapy in two models of anticoagulation service - a prospective randomized trial. $\mathrm{Br} J \mathrm{Clin}$ Pharmacol 2006, 62(5):601-9.

19. Driesen A, Verbeke $K$, Simoens $S$, Laekeman G: International trends in lifelong learning for pharmacists. Am J Pharm Educ 2007, 71(3):52.
20. Dobson RT, Henry CJ, Taylor JG, Zello GA, Lachaine J, Forbes DA, Keegan DL: Interprofessional health care teams: attitudes and environmental factors associated with participation by community pharmacists. J Interprof Care 2006, 20(2):119-32.

21. Kelleher D, Gabe J, Williams G: Understanding medical dominance in the modern world. Challenging Medicine London and New York: Routledge; 1994, xi-xxix.

22. Bradshaw SJ, Doucette WR: Community pharmacists as patient advocates: physician attitudes. J Am Pharm Assoc 1998, 38(5):598-602

23. Edmunds J, Calnan MW: The reprofessionalisation of community pharmacy? An exploration of attitudes to extended roles for community pharmacists amongst pharmacists and General Practioners in the United Kingdom. Soc Sci Med 2001, 53(7):943-55.

24. Hon EKL, Lee K, Tse HM, Lam LN, Tam KC, Chu KM, Lee V, Lau C, Leung TF: A survey of attitudes to traditional Chinese medicine in Hong Kong pharmacy students. Complement Ther Med 2004, 12(1):51-6.

25. Chung VCH, Lau CH, Yeoh EK, Griffiths SM: Age, chronic noncommunicable disease and choice of traditional Chinese and western medicine outpatient services in a Chinese population. BMC Health Serv Res 2009, 9:207.

doi:10.1186/1749-8546-5-41

Cite this article as: Chi Ho Chung et al:: Use of chinese and western over-the-counter medications in Hong Kong. Chinese Medicine 2010 5:41.

\section{Submit your next manuscript to BioMed Central and take full advantage of:}

- Convenient online submission

- Thorough peer review

- No space constraints or color figure charges

- Immediate publication on acceptance

- Inclusion in PubMed, CAS, Scopus and Google Scholar

- Research which is freely available for redistribution
Biomed Central 